\title{
AN ESTIMATED APPROACH OF AMERICAN LOOKBACK OPTIONS PRICE
}

\section{YANAN YUN}

Department of Mathematics

Jinan University

Guangzhou Guangdong 510632

P. R. China

e-mail: yanan_yun@163.com

\begin{abstract}
In this article, we use the least square Monte Carlo approach to estimate American lookback options, and use Sobol sequence and antithetic variable technique to reduce variance. Not only the specific steps of the OSLSM approach are given, but also the numerical examples are given. By comparing option price, standard deviation and price variance, it is found that the OSLSM approach using Sobol sequence and antithetic variable technique can get better simulation results than the LSM method.
\end{abstract}

\section{Introduction}

Exotic options are a new type of options by changing, combining, deriving standard options. Lookback options are a kind of exotic options. On the option maturity, the holder of lookback option can look back on the evolution process of the stock price during the period of validity of the

2020 Mathematics Subject Classification: 97M30, 97M40.

Keywords and phrases: American options, lookback options, antithetic variable technique, Sobol sequence.

Received November 26, 2019; Revised March 7, 2020

(C) 2020 Scientific Advances Publishers 
option, and select the highest (lowest) stock price as the execution price, then, sell (buy) the stock. It is to say that the income of the lookback options depends on the maximum or minimum value that the stock price can reach during the period of validity of option. Thus, lookback options are a kind of strong path related options, also known as "sell at a high price, buy at a low price". According to the concept of lookback options, the option holder can always sell the stock at the highest price or buy the stock at the lowest price during the validity of option. So, the option holder can always be in the most favourable position, which decides the income of lookback options is relatively high. Hence, the price of lookback options is very expensive.

According to the way of exercise, options can be divided into European options and American options. Compared with European options, American options grant the holder the privilege of exercising options in advance. Therefore, the price of American options is related to the best implementation time. In order to obtain the maximum profit, the holder must take the best implementation strategy. The feature that American options can be executed in advance makes it impossible to calculate option prices using the classic Black-Scholes formula [1]. At present, American options are mainly estimated by numerical approaches, such as, the binomial-tree approach [2], the finite differential approach [2], the finite element approach [3], the Monte Carlo approach $[4,5]$ and so on. The Monte Carlo approach has obvious advantages in estimating the price of the path dependent options. In 1993, Tilly [6] used the Monte Carlo approach ( $\mathrm{MC}$ approach) to estimate American put options. Broadie and Glasserman [7] improved MC approach, but the simulation results are poor. Until 2001, Longstaff and Schwartz [4] used the least squares Monte Carlo approach (the LSM approach) to calculate the price of American options, which was widely accepted currently. In order to obtain the price of American options, the LSM approach uses the estimated conditional expectation value of American options to judge whether the path is implemented, and then obtains the optimal exercise point of each path. 
The accuracy of Monte Carlo approach is closely related to the number of simulations, that is to say, the more simulation times, the higher calculation accuracy. However, as the number of simulations increases, the number of computations will increase. To reduce the simulation variance, there are many methods, such as antithetic variable technique [8], control variable technique [8], stratified sampling technique [8] and so on. Pseudo-random number sequence is a finite collection of cycles, and the upper bound of the sequence deviation will be maximized, so that the MC simulation effects are not idea. Therefore, it is important to use the deterministic sequence with low deviation to replace the pseudo-random number sequence. To meet this requirement, there are many sequences have been constructed, such as, Halton sequence [9], Sobol sequence [10], Faure sequence [10], which are called as quasirandom sequences or low deviation sequences. Therefore, some scholars proposed to use the low deviation sequence instead of the pseudo-random sequence in Monte Carlo approach, which is called as Quasi Monte Carlo approach.

In this article, we introduce the generation method and properties of Sobol sequence, then describe that the basic idea of using antithetic variable technique to reduce the variance, finally adopt the improved least squares Monte Carlo method to estimate the price of the American lookback option.

\section{The Introduction of Sobol Sequence}

Sobol sequence is a quasi-random sequence with two as the base, and its generation process is as follows [10].

(1) Any decimal integer can be uniquely decomposed into a formula

with two as the base, i.e., $n=\sum_{i=0}^{m} a_{i} 2^{i}$, where $m$ is the smallest positive integer greater than or equal to $\log _{2} n$, and $a_{i} \in\{0,1\}$. 
(2) Define a $k$ degree primitive polynomial

$$
P=x^{k}+h_{1} x^{k-1}+\cdots+h_{k-1} x+1,
$$

where $h_{j} \in\{0,1\}$. Then we can get $m_{i}$ by recursion formula

$$
m_{i}=2 h_{i-1} \oplus 2^{2} h_{2} m_{i-2} \oplus \cdots \oplus 2^{k-1} h_{k-1} m_{i-k+1} \oplus 2^{k} m_{i-k} \oplus m_{i-d},
$$

where $\oplus$ satisfies the following algorithm: $1 \oplus 0=1,0 \oplus 1=1,0 \oplus 0=0$, $1 \oplus 1=0$.

(3) Define direction number [11] $\nu(i)=\frac{m_{i}}{2^{i}}$, where $m_{i}$ is a positive odd number less than $2^{i}$. The element of the Sobol sequence is

$$
\varphi(n)=a_{1} \nu(2) \oplus a_{2} \nu(3) \oplus \cdots \oplus a_{i} \nu(i) .
$$

The Sobol sequence is obtained by converting $\varphi(n)$ to a decimal number.

(4) Reset $n=n+1$, and then repeat steps (1), (2) and (3).

We use MATLAB software to generate Sobol sequence. Figures 1, 2 and 3 show the properties and the uniform distribution of different dimensions of Sobol sequence. It can be seen from the following three pictures that the distribution uniformity of Sobol sequence is good. There is no high-dimensional degradation of Sobol sequence in the 47-dimensional case. 


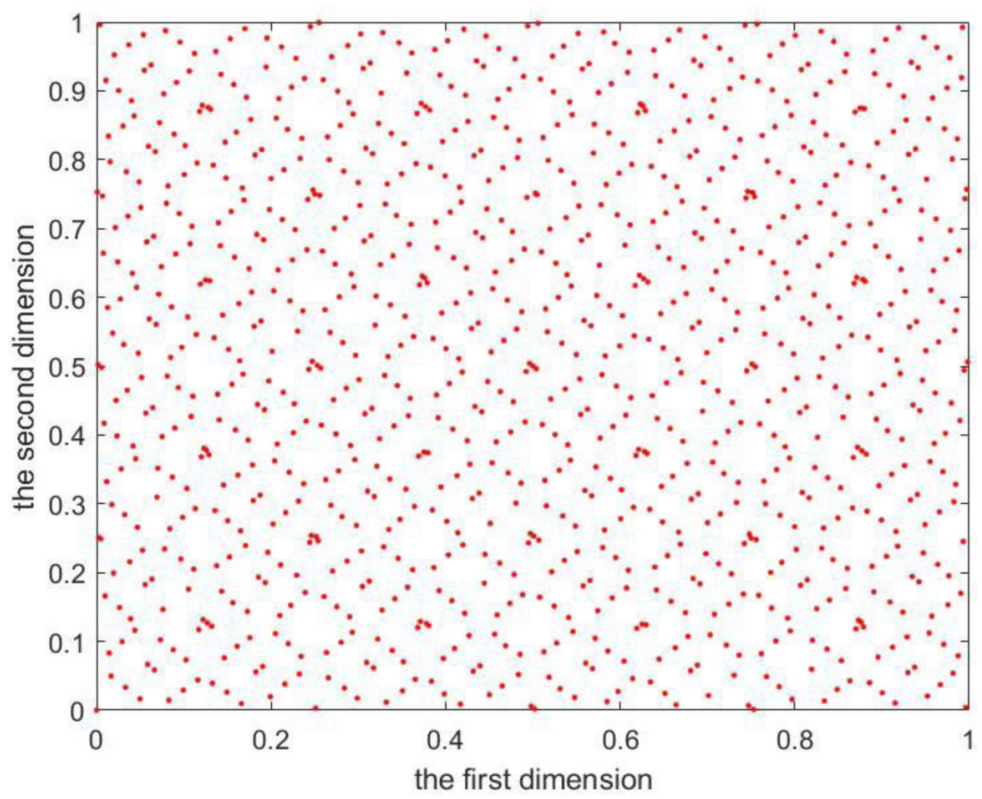

Figure 1. Sobol sequence $(N=1000)$ dimension: $1 \times 2$.

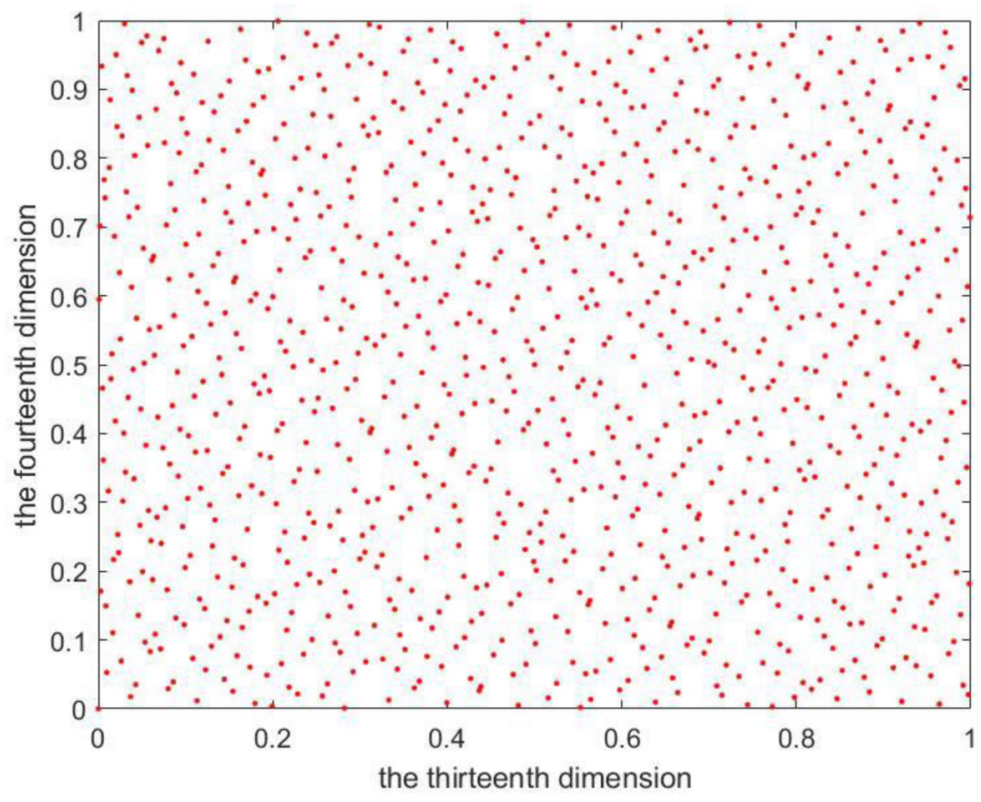

Figure 2. Sobol sequence $(N=1000)$ dimension: $13 \times 14$. 


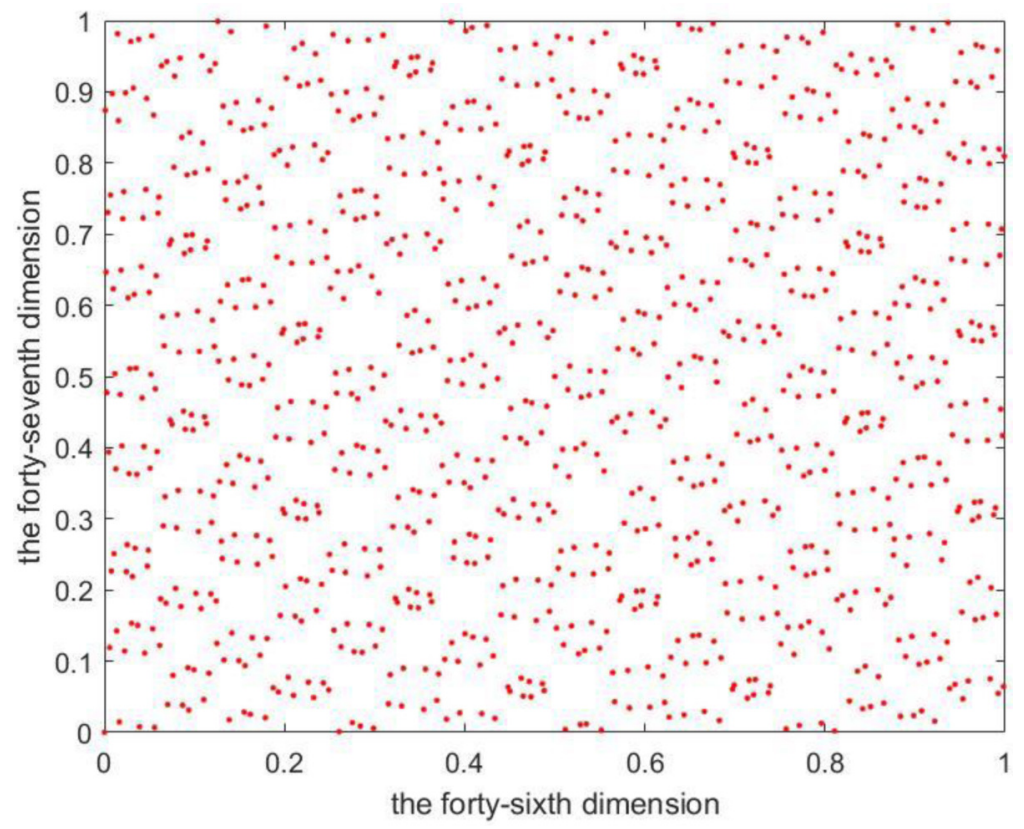

Figure 3. Sobol sequence $(N=1000)$ dimension: $46 \times 47$.

Then we use MATLAB software to compare the statistical indexes of pseudo-random sequence and Sobol sequence (the second dimension), which obeys the standard normal distribution, and drawing the probability density histograms. The results are as follows.

Skewness is a statistic that measures the direction and degree of the deviation of the sample data distribution. Kurtosis is a statistic that measures the characteristics of the tail of the sample data. It can be seen from Table 1 that the statistical values of Sobol sequence are closer to the theoretical values. It can be seen from Figures 4 and 5, Sobol sequence has obvious advantages in fitting the normal distribution skewness and tail characteristics. 
Table 1. Statistical values of pseudo-random sequence and Sobol sequence obeying the standard normal distribution

\begin{tabular}{|c|c|c|c|c|c|}
\hline & \multirow{2}{*}{$\begin{array}{c}\text { Standard } \\
\text { normal } \\
\text { distribution } \\
\begin{array}{c}\text { Theoretical } \\
\text { value }\end{array}\end{array}$} & \multicolumn{2}{|c|}{ Pseudo-random sequence } & \multicolumn{2}{|c|}{$\begin{array}{l}\text { Sobol sequence (the } \\
\text { second dimension) }\end{array}$} \\
\hline & & $\begin{array}{l}\text { Simulation } \\
\text { value }\end{array}$ & Error & $\begin{array}{l}\text { Simulation } \\
\text { value }\end{array}$ & Error \\
\hline Mean & 0 & -0.006827 & 0.006827 & -0.000036 & 0.000036 \\
\hline Variance & 1 & 0.976827 & 0.023173 & 0.999180 & 0.000082 \\
\hline Skewness & 0 & -0.018971 & 0.018971 & -0.000742 & 0.000742 \\
\hline Kurtosis & 3 & 2.991347 & 0.008653 & 2.992763 & 0.007237 \\
\hline
\end{tabular}

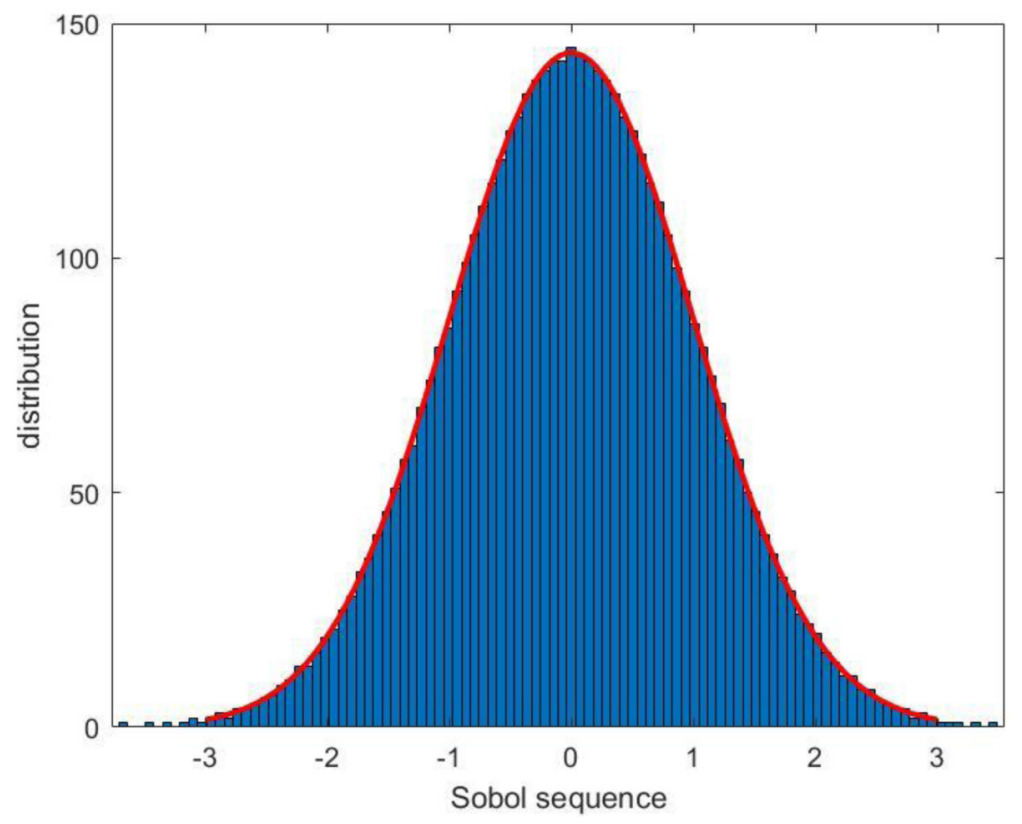

Figure 4. Histogram of Sobol sequence. 


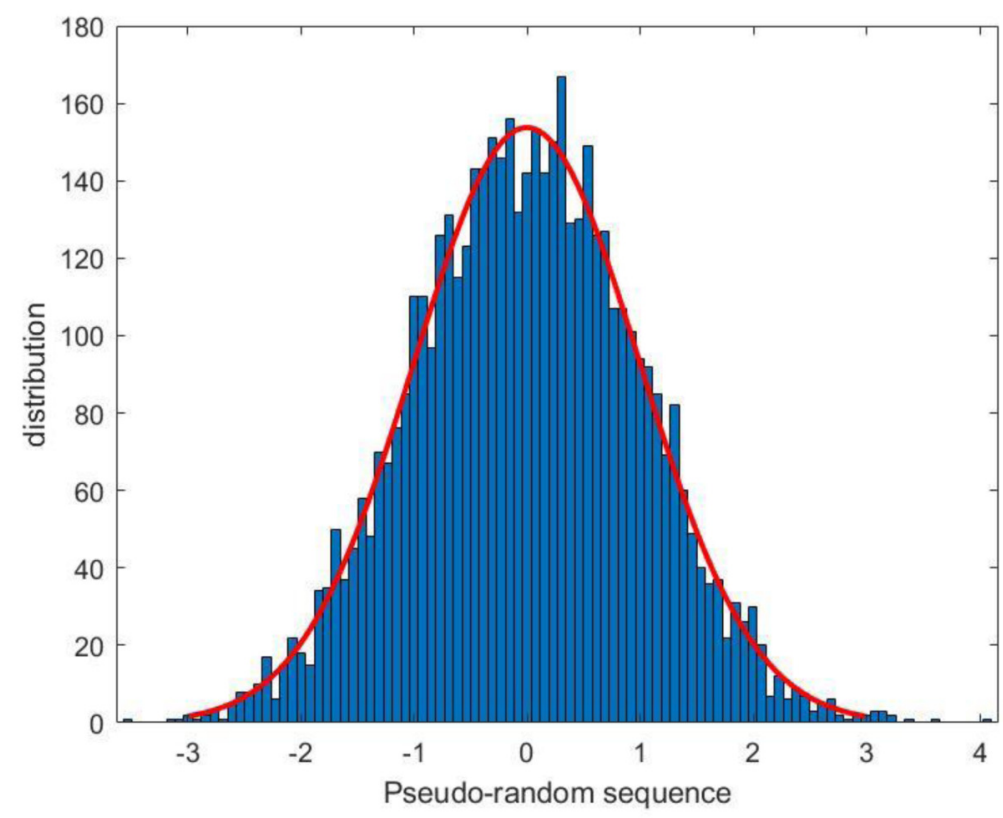

Figure 5. Histogram of pseudo-random sequence.

By comparing Sobol sequence with pseudo-random sequence, it is concluded that the distribution of Sobol sequence has better uniformity and it can better fit the standard normal distribution.

\section{The Introduction of Antithetic Variable Technique}

The antithetic variable technique, which uses the negative correlation between variables to achieve the purpose of reducing variance, is the most common technique to reduce variance. The basic idea of antithetic variable technique is to introduce an antithetic variable, then let the two simulation processes be performed simultaneously in each simulation, finally take the mean of the two simulation results. That is to say, in a simulation process, the first option price $f_{1}$ can be obtained by the usual method, the second option price $f_{2}$ can be obtained by recalculating by changing the sign of all the sample samples in the calculation $f_{1}$.

Therefore, the option price on this simulated path is $f=\frac{1}{2}\left(f_{1}+f_{2}\right)$ [8]. 


\section{The OSLSM Approach for American Lookback Option Price}

According to whether the option execution price is fixed, it can be divided into two types, i.e., American lookback option with the fixed execution price and American lookback option with the floating execution price. The discrete calculation of pricing is

(1) American lookback call option with the floating execution price:

$$
\left\{\begin{aligned}
V_{\text {float-call }}(T)=f_{\text {float-call }}\left(S_{T}\right)=\max \left\{S_{T}-\min _{0 \leq t \leq T} S_{t}, 0\right\}, \\
V_{\text {float-call }}\left(t_{i}, S_{t_{i}}\right)=\max \left\{\max \left\{S_{t_{i}}-\min _{0 \leq s \leq i} S_{t_{s}}, 0\right\},\right. \\
\left.E^{Q}\left[e^{-r \Delta t} V_{\text {float-call }}\left(t_{i+1}, S_{t_{i+1}}\right) \mid S_{t_{i}}\right]\right\} .
\end{aligned}\right.
$$

(2) American lookback put option with the floating execution price:

$$
\left\{\begin{array}{c}
V_{\text {float }- \text { put }}(T)=f_{\text {float }- \text { put }}\left(S_{T}\right)=\max \left\{\max _{0 \leq t \leq T} S_{t}-S_{T}, 0\right\}, \\
V_{\text {float-put }}\left(t_{i}, S_{t_{i}}\right)=\max \left\{\max \left\{\max _{0 \leq s \leq i} S_{t_{s}}-S_{t_{i}}, 0\right\},\right. \\
\left.E^{Q}\left[e^{-r \Delta t} V_{\text {float }- \text { put }}\left(t_{i+1}, S_{t_{i+1}}\right) \mid S_{t_{i}}\right]\right\} .
\end{array}\right.
$$

(3) American lookback call option with the fixed execution price:

$$
\left\{\begin{array}{c}
V_{\text {fixed-call }}(T)=f_{\text {fixed-call }}\left(S_{T}\right)=\max \left\{\max _{0 \leq t \leq T} S_{t}-K, 0\right\}, \\
V_{\text {fixed-call }}\left(t_{i}, S_{t_{i}}\right)=\max \left\{\max \left\{\max _{0 \leq s \leq i} S_{t_{s}}-K, 0\right\},\right. \\
\left.E^{Q}\left[e^{-r \Delta t} V_{\text {fixed-call }}\left(t_{i+1}, S_{t_{i+1}}\right) \mid S_{t_{i}}\right]\right\} .
\end{array}\right.
$$


(4) American lookback put option with the fixed execution price:

$$
\left\{\begin{array}{l}
V_{\text {fixed-put }}(T)=f_{\text {fixed-put }}\left(S_{T}\right)=\max \left\{K-\min _{0 \leq t \leq T} S_{t}, 0\right\}, \\
V_{\text {fixed-put }}\left(t_{i}, S_{t_{i}}\right)=\max \left\{\max \left\{K-\min _{0 \leq s \leq i} S_{t_{s}}, 0\right\},\right. \\
\left.E^{Q}\left[e^{-r \Delta t} V_{\text {fixed-put }}\left(t_{i+1}, S_{t_{i+1}}\right) \mid S_{t_{i}}\right]\right\} .
\end{array}\right.
$$

Taking American lookback call option with the floating execution price for example, the specific steps of the OSLSM approach are as follows. The calculation method of other types of option price is similar.

Step 1. Generating a random Sobol sequence

$$
\varepsilon=\left(\varepsilon_{i \times j}\right), i=1,2, \cdots, n ; j=1,2, \cdots, N .
$$

First, generating Sobol sequence $s$, and using the rand function in MATLAB to generate random number $u$ obeyed to uniform distribution. Therefore, $x=\{s+u \bmod 1\}$ is a random Sobol sequence. Finally, by inverting the cumulative normal distribution of $x$, so a random Sobol sequence $\varepsilon=\left(\varepsilon_{i \times j}\right)$ that obeys the standard normal distribution can be obtained.

Step 2. Simulating the changing path of the stock price process $\left\{S_{t}, 0 \leq t \leq T\right\}$.

Under the risk neutrality measure, assuming the stock price process $\left\{S_{t}, 0 \leq t \leq T\right\}$ is obey the following stochastic differential equation:

$$
d S_{t}=r S_{t} d t+\sigma S_{t} d W_{t}
$$

where $r$ is the risk-free rate, $\sigma$ is the volatility of the stock price, and $\left\{W_{t}, 0 \leq t \leq T\right\}$ is a standard Brownian motion. Assume that the stock price at the initial moment is $S_{0}$, the option term $T$ is divided into $N$ 
equal parts, that is, $\Delta t=\frac{T}{N}, 0=t_{0}<t_{1}<\cdots<t_{N}=T$, the simulation path is $M$, thus the stock price matrix can be obtained $S_{(N+1) \times M}$, where $S_{(i+1), j}=S_{i, j} \exp \left\{\left(r-\frac{1}{2} \sigma^{2}\right) \Delta t+\sigma \varepsilon_{i, j} \sqrt{\Delta t}\right\}(i=0,1, \cdots, N ; j=1,2, \cdots, M)$,

$\varepsilon_{i, j} \in \varepsilon$ are generated by Step 1 .

Taking the opposite number $-\varepsilon_{i, j}$ of the random variable $\varepsilon_{i, j}$ as the antithetic random numbers, then the antithetic matrix $S_{(N+1) \times M}^{*}$ of the stock price is obtained.

Step 3. Calculating the execution time and option value at time $t_{N-1}$.

(1) At time $T$, calculating the immediate strike value $f_{N, j}=\max \left\{\max _{0 \leq i \leq N} S_{i, j}-S_{N, j}, 0\right\}$ of options in each path, so the cash flow generated by option trading at time $T$ is $C_{N, j}=f_{N, j}$.

(2) Calculating the immediate strike value $f_{N-1, j}=\max \left\{\max _{0 \leq i \leq N-1}\right.$ $\left.S_{i, j}-S_{N-1, j}, 0\right\}$ of options in each path at time $t_{N-1}$, and finding the path of $f_{N-1, j^{\prime}}>0$, using the least square method to calculate the intrinsic estimated value $F_{N-1, j^{\prime}}$ of option to approximate the conditional expected value $E\left[e^{-r \Delta t} f_{N, j^{\prime}} \mid S_{N-1, j^{\prime}}\right]$.

(3) Comparing the intrinsic estimated value $F_{N-1, j^{\prime}}$ with the strike value $f_{N-1, j^{\prime}}$ to determine whether option is exercised at the current moment. If $F_{N-1, j^{\prime}}<f_{N-1, j^{\prime}}$, the option should be executed immediately, so $t_{N-1}$ is an execution time, which is denoted as $\tau_{j^{\prime}}=t_{N-1}$, the cash flow at the current moment is $C_{N-1, j^{\prime}}=f_{N-1, j^{\prime}}$, and the cash flow at 
time $T$ is $C_{N, j^{\prime}}=0$. On the contrary, we should continue to hold the option to execute at time $T$. Then the cash flow at the current moment is $C_{N-1, j^{\prime}}=0$, and the cash flow at time $T$ is $C_{N, j^{\prime}}=f_{N, j^{\prime}}$.

(4) For the path of $f_{N-1, j^{\prime \prime}}=0$, the execution time is $\tau_{j^{\prime \prime}}=T$, and the cash flow at time $t_{N-1}$ is $C_{N-1, j^{\prime \prime}}=0$, and the cash flow at time $T$ is $C_{N, j^{\prime \prime}}=f_{N, j^{\prime \prime}}$

(5) Similarly, the execution time and the cash flow of the antithetic sample can be updated.

Step 4. Calculating the execution time and option value at time $t_{i}(i=n-2, \cdots, 2,1)$.

Repeating Step 3 to obtain the cash flow and execution strategy at time $t_{i}(i=N-2, N-1, \cdots, 1)$ of each path, and updating the optimal stop time $\tau=\left(\tau_{j}\right)_{j=1}^{M}$. Similarly, the optimal stop time $\tau^{*}=\left(\tau_{j^{*}}\right)_{j^{*}=1}^{M}$ of the dual path can be obtained.

Step 5. Calculating the estimated value of American lookback option. The price of the option can be obtained by discounting the cash flow of all paths at the optimal stop to the initial moment and then averaging all paths, that is

$$
V_{0}=\frac{1}{2 N}\left[\sum_{j=1}^{N} e^{-r \tau_{j}} C\left(\tau_{j}\right)+\sum_{j=1}^{N} e^{-r \tau_{j^{*}}} C\left(\tau_{j^{*}}\right)\right] .
$$

\section{Numerical Analysis}

Considering American lookback call option with floating strike price, the initial price of the underlying stock $s 0=100$, the risk-free interest rate $r=0.1$, the volatility $=0.3$, the maturity date $T=0.5$. During the period of validity, option can be executed 128 times, and the number of simulated paths being $200,400,600,800$, and 1000, respectively. In order 
to compare the effect of variance reduction, we respectively use the LSM approach, the SLSM approach (combined with Sobol sequence), the OLSM approach (combined with antithetic variable technique) and the OSLSM approach (combined with Sobol sequence and antithetic variable technique) to calculate option price. Description: under a certain number of simulation paths, using four methods to simulate 100 times respectively, taking the average of option price obtained in 100 simulations as the final price, using the mean of standard deviation of each simulation as standard deviation of all simulations, and letting price variance of 100 simulations to measure volatility.

Table 2. Under the floating execution price, using the LSM approach and the SLSM approach estimate the price of American lookback call option

\begin{tabular}{ccccccc}
\hline \multirow{2}{*}{$\begin{array}{c}\text { Number of } \\
\text { simulations }\end{array}$} & $\begin{array}{c}\text { Option } \\
\text { price }\end{array}$ & $\begin{array}{c}\text { Standard } \\
\text { deviation }\end{array}$ & Volatility & $\begin{array}{c}\text { Option } \\
\text { price }\end{array}$ & $\begin{array}{c}\text { Standard } \\
\text { deviation }\end{array}$ & Volatility \\
\hline 200 & 9.67768 & 6.402147 & 0.217951 & 9.60036 & 5.391344 & 0.243811 \\
400 & 9.54899 & 6.183667 & 0.102860 & 9.48383 & 5.078175 & 0.135572 \\
600 & 9.50116 & 5.920129 & 0.081864 & 9.46641 & 4.936575 & 0.075032 \\
800 & 9.44004 & 5.786834 & 0.051595 & 9.43895 & 4.835523 & 0.057251 \\
1000 & 9.43532 & 5.803918 & 0.051914 & 9.45016 & 4.826593 & 0.033257 \\
\hline
\end{tabular}

Table 3. Under the floating execution price, using the OLSM approach and the OSLSM approach estimate the price of American lookback call option

\begin{tabular}{|c|c|c|c|c|c|c|}
\hline \multirow{2}{*}{$\begin{array}{l}\text { Number of } \\
\text { simulations }\end{array}$} & \multicolumn{3}{|c|}{ OLSM approach } & \multicolumn{3}{|c|}{ OSLSM approach } \\
\hline & $\begin{array}{l}\text { Option } \\
\text { price }\end{array}$ & $\begin{array}{l}\text { Standard } \\
\text { deviation }\end{array}$ & Volatility & $\begin{array}{l}\text { Option } \\
\text { price }\end{array}$ & $\begin{array}{l}\text { Standard } \\
\text { deviation }\end{array}$ & Volatility \\
\hline 200 & 9.65934 & 5.556053 & 0.088233 & 9.67935 & 4.494469 & 0.102922 \\
\hline 400 & 9.53481 & 5.183212 & 0.043582 & 9.51072 & 4.178793 & 0.039436 \\
\hline 600 & 9.48477 & 4.980582 & 0.028262 & 9.47783 & 3.997898 & 0.027220 \\
\hline 800 & 9.44775 & 4.869866 & 0.021527 & 9.43702 & 3.898191 & 0.019963 \\
\hline 1000 & 9.41082 & 4.794161 & 0.022909 & 9.40870 & 3.793280 & 0.013669 \\
\hline
\end{tabular}


By comparing the data in Table 2 and Table 3, conclusions are as follows:

(1) Under the condition of a certain number of simulation paths, the results obtained by four simulation algorithms are not significantly different. The variance and volatility of option price obtained by the OSLSM approach are smaller than the other three methods, so the simulation results of the OSLSM approach are relatively stable.

(2) With the increase of the number of simulation paths, four simulation algorithms can reduce the variance and the price volatility correspondingly. The OSLSM approach has the most obvious effect on reducing the variance and price volatility, the OLSM approach and the SLSM approach followed, and the LSM approach is the worst.

(3) By observing the data of the column of standard deviation, it can be found that the OSLSM approach can obtain the same result of variance reduction as the LSM approach under the condition of fewer simulation paths.

(4) The results obtained by using the OSLSM approach are more stable than those obtained by using the LSM approach, which indicates that the variance of simulation results can be reduced by using relatively uniform Sobol sequence. The simulation results are more stable due to its feature of low sequence difference, which reduces the calculation amount to some extent, at the same time, more accurate approximate estimation of option price can be obtained.

By analyzing the data in Table 2 and Table 3, it can be found that the OSLSM approach is superior to the LSM approach in the effect of the variance reduction and the stability of the calculated results. It suggests that the better simulation results can be obtained by using the OSLSM approach for American lookback call options. 


\section{References}

[1] F. Black and M. Scholes, The pricing of options and corporate liabilities, Journal of Political Economy 81(3) (1973), 637-654.

[2] John C. Hull, Options, Futures, and Other Derivatives, (Sixth Edition), The People's Posts and Telecommunications Press, Beijing, 2010.

[3] Haiming Song, Qi Zhang, Jingzhi Li and Hongyu Liu, Finite element method for valuation of American lookback options, Mathematica Numerica Sinica 38(3) (2016), 245-256.

[4] F. A. Longstaff and E. S. Schwartz, Valuing American options by simulation: A simple least-squares approach, Review of Financial Studies 14(1) (2001), 113-147.

DOI: https://doi.org/10.1093/rfs/14.1.113

[5] Cheng-Li Zheng, Comparison of three kinds of Monte Carlo methods for American option pricing, Journal of System Simulation 18(10) (2006), 2929-2935.

[6] J. A. Tilly, Valuing American options in a path simulation model, Transactions of the Society of Actuaries 45 (1993), 55-67.

[7] M. Broadie and P. Glasserman, Pricing American-style securities using simulation, Journal of Economics Dynamics and Control 21(8-9) (1997), 1323-1352.

DOI: https://doi.org/10.1016/S0165-1889(97)00029-8

[8] Zhiyong Zheng, Financial Quantity Analysis Based on MATLAB Programming, (Third Edition), Beihang University Press, Beijing, 2014.

[9] J. H. Halton, On the efficiency of certain quasi-random sequences of points in evaluation multi-dimensional integrals, Numerische Mathematik 2 (1960), 84-90.

DOI: https://doi.org/10.1007/BF01386213

[10] Fuyan Luo and Haiyun Xu, The applying of quasi-Monte Carlo methods in financial computation, Application of Statistics and Management 27(4) (2008), 605-610.

[11] V. Lakshmikantham, S. K. Sen and T. Samanta, Comparing random number generators using Monte Carlo integration, International Journal of Innovative Computing, Information and Control 1(2) (2005), 143-165. 